\title{
Study of Stimulus-Induced Plasticity in Neural Networks Cultured in Microftuidic Chips
}

DOI: $10.17691 / \mathrm{stm} 2017.9 .4 .02$

Received March 22, 2017

A.A. Gladkov, Junior Researcher, Laboratory of Neuroengineering, Center for Translational Technologies ${ }^{1}$ :

Junior Researcher, Molecular and Cellular Technologies Department, Central Research Laboratory2;

V.N. Kolpakov, Research Assistant, Laboratory of Neuroengineering, Center for Translational Technologies'; Y.I. Pigareva, Junior Researcher, Laboratory of Neuroengineering, Center for Translational Technologies:;

A.S. Bukatin, PhD, Researcher, Laboratory for Nanobiotechnologies;

V.B. Kazantsev, DSc, Vice-Rector for Research ${ }^{1}$; Head of the Department of Neurotechnologies, Institute of Biology and Biomedicine ${ }^{1}$; Head of the Laboratory for the Development of Intellectual Biomechatronic Technologies, Center for Translational Technologies ${ }^{1}$;

I.V. Mukhina, DSc, Professor, Head of the Center for Translational Technologies';

Head of the Central Research Laboratory2; Head of the Department of Normal Physiology

named after N.Y. Belenkov2; Professor, Neurotechnology Department, Institute of Biology and Biomedicine ${ }^{1}$;

A.S. Pimashkin, PhD, Head of the Laboratory of Neuroengineering, Center for Translational Technologies ${ }^{1}$

${ }^{1}$ Lobachevsky State University of Nizhni Novgorod, 23 Prospekt Gagarina, Nizhny Novgorod, 603950,

Russian Federation;

${ }^{2}$ Nizhny Novgorod State Medical Academy, 10/1 Minin and Pozharsky Square, Nizhny Novgorod, 603005,

Russian Federation;

${ }^{3}$ Saint Petersburg National Research Academic University of the Russian Academy of Sciences, 8/3 Khlopina St.,

Saint Petersburg, 194021, Russian Federation

Dissociated brain cell cultures on microelectrode arrays are widely used to study fundamental mechanisms of information processing and synaptic plasticity. It has been established that high frequency electrical stimulation causes functional changes in neural networks. However, complex and homogeneous morphological structure of cultured brain cell networks presents a significant challenge for further evaluation of the synaptic plasticity at the network level. In this study, we propose a new approach to studying neural network plasticity using microfluidic devices with specially designed channels. Microfluidic chips can guide axons and form neural circuits with two subnetworks connected by synaptic paths in the required direction. To induce synaptic plasticity, high frequency tetanic stimulation by two groups of electrodes located in the area of pre- and postsynaptic neurons was applied. The developed method of potentiation and depression of the required functional connectivity in the neural circuit can be used to further study network effects of synaptic plasticity induced in the local subpopulation of cells.

Key words: neural networks in vitro; microfluidics; neuroengineering; synaptic plasticity; spike timing dependent plasticity; STDP; information processing by the brain.

Dissociated neural cell cultures are widely used as an experimental model for long-term studies of synaptic plasticity in neuronal networks, learning and memory [1-10]. This model makes it possible to carry out longterm monitoring of bioelectrical functional activity and morphological changes in neural networks. There are a significant number of studies devoted to activity-induced plasticity in dissociated brain cell networks cultured for a long time on microelectrode arrays. The most common electrical stimulation protocols are closedloop stimulation (stimulation depending on network responses) [1-4], low-frequency stimulation $(1-0.5 \mathrm{~Hz})$ $[5,6]$ and high frequency tetanic stimulation [7-10] based on spike timing dependent plasticity (STDP).
In dissociated neural cell networks, connections between neurons are formed randomly. To reveal functional interconnections and their changes after stimulation, various methods of mathematical analysis are needed $[11,12]$. In recent years, development of microfluidic techniques has allowed guiding growth of neurites and forming cultures with required functional connections [13-20]. Connections in which signals propagate in one direction are the most interesting because pre- and postsynaptic cells involved in them are in separate chambers. Therefore, it is convenient to use stimulation based on STDP. We have investigated signal propagation through axons between two populations of neural cells after high-frequency stimulation with a

For contacts: Arseniy A. Gladkov, e-mail: gladkov@neuro.nnov.ru 
delay in accordance with STDP. Changes in propagation of responses to stimuli have been observed after stimulation. This suggests that the developed approach is effective for induction of plasticity by electrical stimuli in dissociated neural cell cultures.

\section{Materials and Methods}

Microfluidic chip fabrication. To create directed synaptic connections, we developed a microfluidic chip with specifically designed microchannels connecting two chambers (Figure 1). The shape of the microchannels (Figure 1 (b)) guides axonal growth predominantly from one chamber - the source chamber (chamber A) into the receiver chamber (chamber $B$ ). Microchannels between the chambers consist of a sequence of triangular segments. Unidirectional connectivity is achieved through the use of "guideways" on the side walls, which limit possible directions of axonal growth and prevent axons from growing in the opposite direction by means of a bottleneck. A microfluidic chip is fabricated by soft-lithography methods using polydimethylsiloxane (PDMS) (Figure 1 (c)).

For master mold fabrication, standard two-layer optical lithography was used: the silicon substrate was coated with the first $4.5 \mu \mathrm{m}$ thick layer of SU-8 2025 negative photoresist, diluted with $\mathrm{T}$ thinner solvent (MicroChem, USA), the second $200 \mu \mathrm{m}$ thick layer of SU-8 2075 was applied after the exposure. Heat treatment at $95^{\circ} \mathrm{C}$ was performed for 4 and $7 \mathrm{~min}$ for the first and second layer, respectively. Exposure was performed through a chrome photomask using MJB4 device (SUSS MicroTec, Germany) with PL-360-LP UV-filter (Omega Optics, USA). Next, exposure silicon substrates were annealed at $95^{\circ} \mathrm{C}$ for 5 and $7 \mathrm{~min}$, respectively. Subsequently, master molds were subjected to heat treatment at $200^{\circ} \mathrm{C}$ for $7 \mathrm{~min}$.

Two components, Sylgard 184 silicone elastomer base and Sylgard 184 silicone elastomer curing agent
(DowCorning, USA), were mixed in the proportion of 10:1 to fabricate PDMS microchips. Uncured PDMS was degassed in a desiccator, poured into the master mold, and then cured at $70^{\circ} \mathrm{C}$ for $4 \mathrm{~h}$. Then PDMS chips were removed from the master molds and holes were punched at the chamber sites of the chips. Each PDMS chip was positioned in the preferred way and then mounted on the surface of the microelectrode array (MEA) (Figure 1 (a)). Before the cells were plated on MEA, its surface had been pre-coated with polyethylenimine molecules (Sigma P3143, USA) to promote cell adhesion.

Cell culture. Hippocampal cells were dissociated from embryonic mice (E18) and plated on PDMS chip chambers of MEA pre-treated with polyethylenimine molecules at final density of $8,000-10,000$ cells $/ \mathrm{mm}^{2}$ [3]. C57B1/6 mice (pregnant females) were sacrificed by cervical dislocation in accordance with the protocols for the care and use of laboratory animals approved by the Ministry of Health of the Russian Federation, the Ethics Committee of Nizhny Novgorod State Medical Academy. Embryos were removed from the uterus and decapitated. The hippocampi separated from the cortex, cerebellum and brain stem were dissected under sterile conditions in phosphate-buffered saline. After enzymatic degradation at $35.5^{\circ} \mathrm{C}$ in $0.25 \%$ trypsin (Invitrogen 25200-056) for $20 \mathrm{~min}$, the cells were suspended using $1 \mathrm{~mm}$ diameter pipette tip. Next, the solution was centrifuged at $1,000 \mathrm{rpm} / \mathrm{min}$ for $4 \mathrm{~min}$. The precipitated cells were immediately re-suspended in Neurobasal medium (Invitrogen 21103-049) enriched with 2\% Supplement B-27 (Invitrogen 17504-044), glutamine (Invitrogen 25030-024), 5\% fetal calf serum (PanEco K055) and gentamicin. Dissociated cells in an $8 \mu$ droplet were placed in PDMS chip chambers. The cells precipitated and formed a dense monolayer on the electrode area of MEA. Once the cells attached (usually within $15 \mathrm{~min}$ ), the dishes were filled with $1 \mathrm{ml}$ Neurobasal medium with $2 \%$

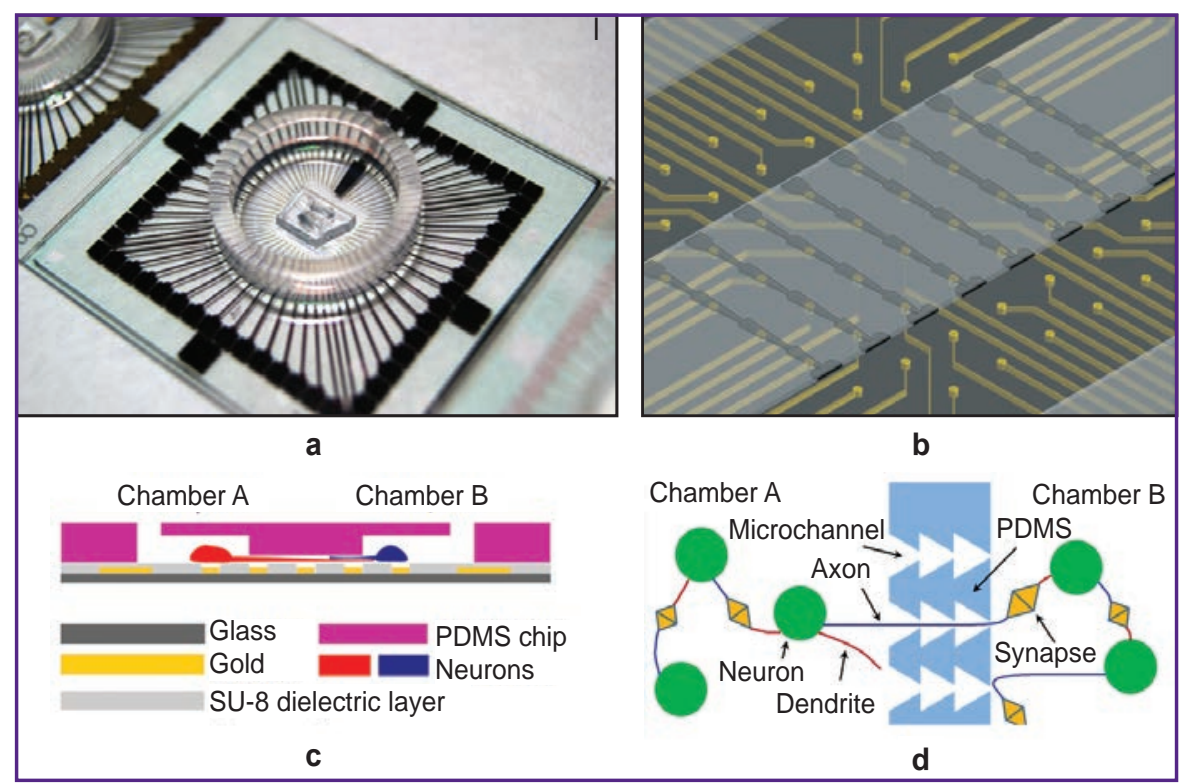

Figure 1. Experimental model of dissociated hippocampal cell culture on a microfluidic device:

(a) a microelectrode array with a microfluidic PDMS chip; (b) schematic arrangement of microfluidic chips on the electrode array; 8 microchannels $(600 \mu \mathrm{m})$ connect two chambers; (c) the front view of the microfluidic chip attached to the microelectrode array; two neuronal subcultures connected with each other by axons in microchannels; (d) specific design of microchannels provides unidirectional growth of neurites 
B-27, 0.5\% glutamine and 5\% fetal calf serum. $24 \mathrm{~h}$ later, half of the medium was replaced by Neurobasal medium containing $1 \%$ glutamine, $2 \%$ B-27 and $0.4 \%$ fetal calf serum, but without antibiotics. Glial growth was not suppressed since glial cells were necessary to preserve culture viability for a long time. Half of the medium was replaced every 2 days. Cells were cultured under constant conditions: $35.5^{\circ} \mathrm{C}, 5 \% \mathrm{CO}_{2}$ in a humidified incubator for cell cultures (MCO-18AIC; SANYO Electric Co., Japan).

Phase-contrast images of cultures were obtained weekly to register the status of cultures using Leica DMIL HC inverted microscope (Leica Microsystems, Germany) with 10x/0.2Ph1 objective lens. The experiments were performed after culturing the neural networks for 3-5 weeks. Five cultures were used.

Electrophysiological activity stimulation and recording. Bioelectrical activity of cultures was recorded 20 days after plating in vitro using USB MEA120-2-Inv-BC system (Multichannel Systems, Germany).

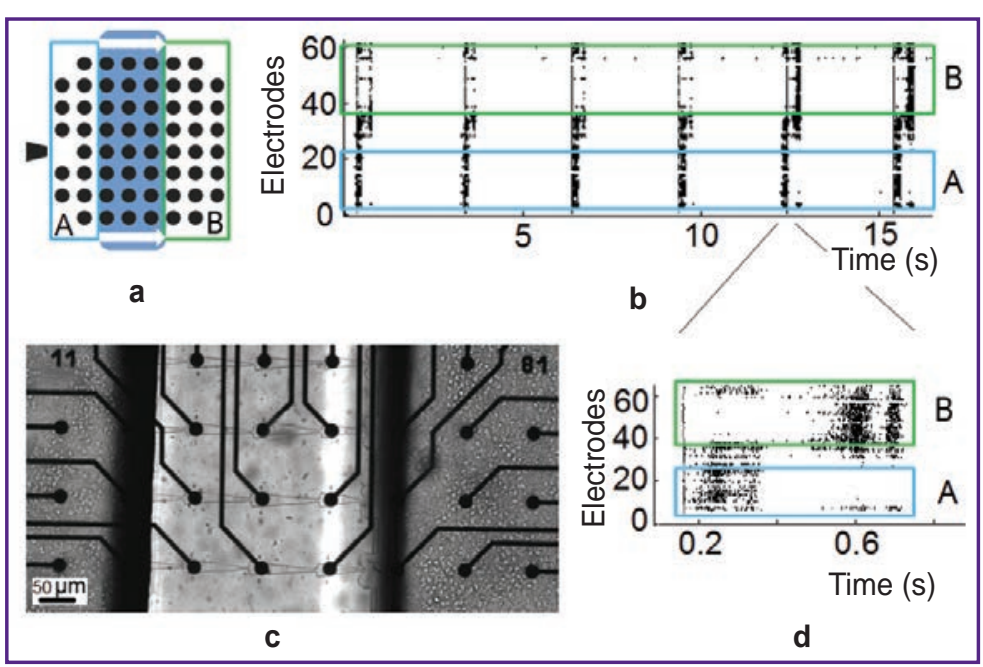

Figure 2. Network activity recording:

(a) the diagram of a microelectrode array with a microfluidic chip; chamber A with presynaptic (green) and chamber B with postsynaptic (blue) neurons connected by microchannels; (b) raster plots of stimulus responses; (c) hippocampal cells cultured on a microelectrode array with 60 electrodes in the microfluidic chip with two chambers for cell bodies and microchannels for neuritis; (d) raster plot of the signal transmitted from chamber $A$ to chamber $B$ after stimulation of chamber $A$
Signals were recorded on MEA with 59 planar electrodes of $30 \mu \mathrm{m}$ diameter and $200 \mu \mathrm{m}$ interelectrode distance. Electrical stimulation was performed using STG-4004 stimulator (Multichannel Systems, Germany). Bioelectrical spikes were detected separately for each channel using threshold detection method [21] developed earlier. The amplitudes of bioelectrical spikes were in the range of $10-80 \mu \mathrm{V}$. Statistic analysis of signals was performed using custom-made software developed in MATLAB [3]. Bioelectrical spikes recorded on the electrodes and corresponding to neuron grown in separate chambers A and B were analyzed separately (Figure 2).

Stimulation protocol. Electrical stimulation consisted of bi-phasic pulses of $\pm 800 \mathrm{mV}$ voltage and $600 \mu \mathrm{s}$ duration. It involved low-frequency control stimulation to evoke and evaluate the network responses and high-frequency tetanic stimulation to induce synaptic plasticity. Control stimulation was performed using three different electrodes in chamber $A$ and consisted of 60 stimuli for each electrode with a 3-second interval between stimuli. Tetanic stimulation was applied using two sets of electrodes: 4 "presynaptic" electrodes in chamber A and 4 "postsynaptic" electrodes in chamber B (Figure 3). The protocol was based on STDP property [8, 9] and consisted of 150 sequences of 20 stimuli with a 100-ms interval between pulses and a 6-second interval between the sequences. To induce potentiation of neurons connected and contained in chambers $A$ and $B$, the delay between "presynaptic" and "postsynaptic" electrodes in tetanic stimulation was $10 \mathrm{~ms}$.

Analysis of evoked responses. Stimulation of each electrode induced a response in the form of a

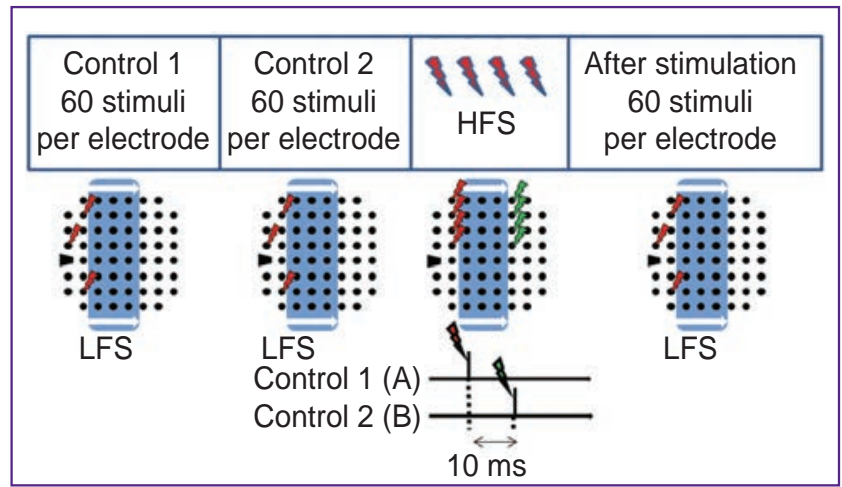

Figure 3. Experimental protocol of stimulation: two control sequences of low-frequency stimulation (LFS) through electrodes in chamber A (60 stimuli per electrode), highfrequency tetanic stimulation (HFS) using STDP protocol and post-tetanic stimulation (see "Materials and Methods")

burst of bioelectrical spikes in a group of electrodes. Raster plots of responses are shown in Figure 2 (b). To characterize the induced bursts, we used poststimulus time histogram (PSTH) $[1,10]$. For each 20-ms interval in the response to stimuli, we calculated total number of electrical spikes recorded at each electrode. PSTH values on the electrodes of chamber A (PSTH A) and chamber B (PSTH B) were analyzed separately (Figure 4).

To evaluate the propagation of bursts evoked by electrical bi-phasic stimuli $( \pm 800 \mathrm{mV}, 260 \mu$ s per phase, the first phase positive, 3-ms intervals between the 


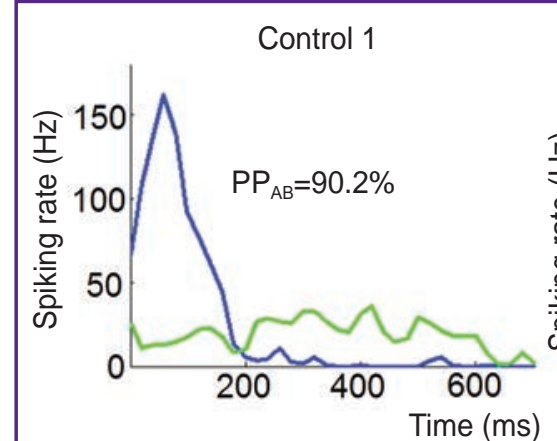

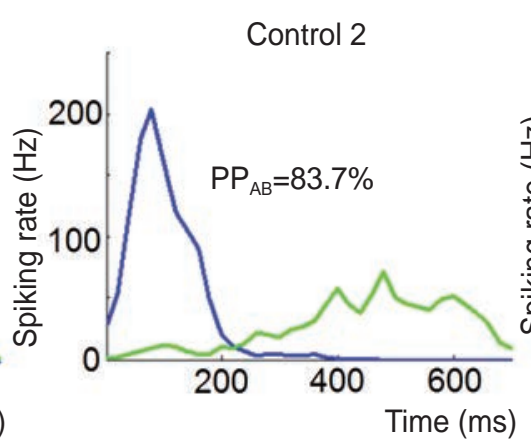

b

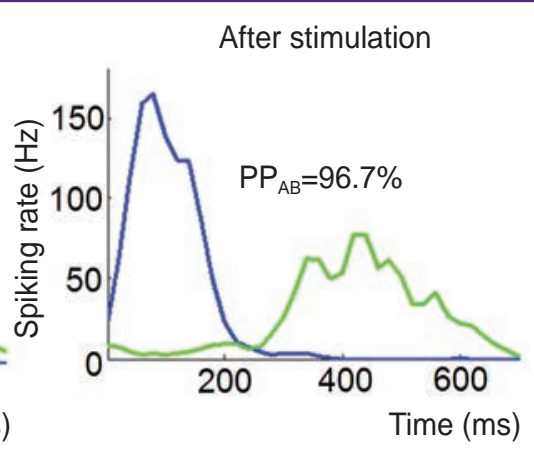

c

Response profile in chamber $A$

Response profile in chamber $B$

Figure 4. Spiking rate in bursts evoked by low-frequency electrical stimulation in presynaptic chamber A (blue curve) and postsynaptic chamber B (green curve) in two control (a), (b) and post-tetanic (c) stimulation sequences. $\mathrm{PP}_{\mathrm{AB}}$ indicates percentage of evoked responses transmitted from presynaptic to postsynaptic subnetwork. $\mathrm{PP}_{\mathrm{AB}}$ increase after tetanic stimulation indicates potentiation of synaptic pathways between neurons in chambers $A$ and $B$

stimuli), a sequence of 60 stimuli was applied through one electrode selected in chamber $A$. The network response was recorded in the range of 10-600 ms after the stimulus. The probability of network bursts evoked on the electrode in chamber A propagating from chamber $A$ to chamber $\mathrm{B}\left(\mathrm{PP}_{\mathrm{AB}}\right)$ was evaluated as a percentage of bursts induced in chamber $A$ which evoked bursts in chamber $B$ of the total number of bursts induced by stimulation in chamber A. Spontaneously generated bursts in non-stimulated chamber can sporadically get into the time interval corresponding to stimulation response.

To test the hypothesis that the bursts observed in chamber B after stimulation reflect real directed propagation of network activity rather than sporadic emergence of spontaneously generated bursts in nonstimulated chamber, analysis with the use of surrogate data was carried out.

We generated surrogate data on bursts in chamber B keeping the number of bursts, their duration and intervals between bursts as in the initial data. Then, the number of post-stimulus spikes (from 10 to $600 \mathrm{~ms}$ ) at 20-ms intervals was calculated. If the number of post-stimulus spikes in chamber B at 20-ms intervals was higher in the initial data than the standard deviation multiplied by 5 in the surrogate data, the observed network activity propagation was considered to be directed.

Only those experimental data in which $\mathrm{PP}_{\mathrm{AB}}$ values exceeded the values calculated for surrogate data (the cultures in which propagation of bursts between the two subnetworks was observed) were selected for further analysis.

\section{Results}

Cultures in the microfluidic chip. PDMS microfluidic chips contained 8 microchannels of $600 \mu \mathrm{m}$ length with four $8 \mu \mathrm{m}$ bottlenecks [22]. Microchannel height equaled $4.5 \mu \mathrm{m}$, which allowed only neuron processes rather than cell bodies to penetrate the channels (see Figure 1 (b), (c)). A specific design was developed to ensure neurite growth in one predominant direction from chamber A to chamber B (Figure 1 (d)). PDMS chip was positioned on the commercial MEA so that 13 electrodes were in chamber A, 24 electrodes on channel sites and 22 electrodes in chamber B (Figure 1 (a)). The arrangement of the chip parts on the electrodes is shown in Figures 1 (b) and 2 (a). Dissociated hippocampal cells (E18) were cultured in both chambers (see "Materials and Methods").

After several days, two subpopulations were connected by neurites via micro-channels (Figure 2 (c)). After 3 weeks, in vitro spontaneous network burst activity propagated predominantly in one direction from chamber A to chamber B. Biphasic electric stimulus $( \pm 800 \mathrm{mV}$, $260 \mu \mathrm{s}$, the first phase positive) through the electrode in chamber A initiated network responses passing from chamber A to chamber B (Figure 2 (b), (d)). The properties of this signal propagation in response to the stimulus were used as indicator of functional connectivity between the two chambers.

Experimental protocol consisted of two control sequences of low-frequency stimulation of 3 electrodes in chamber A (60 stimuli per electrode with a 3-second interval between the stimuli) to evaluate the stability of stimulus response characteristics and a sequence of low-frequency stimulation of the same electrodes after tetanic stimulation (see Figure 3).

Axons grown through microchannels formed synaptic contacts with the nearest neurons in chamber $B$, 


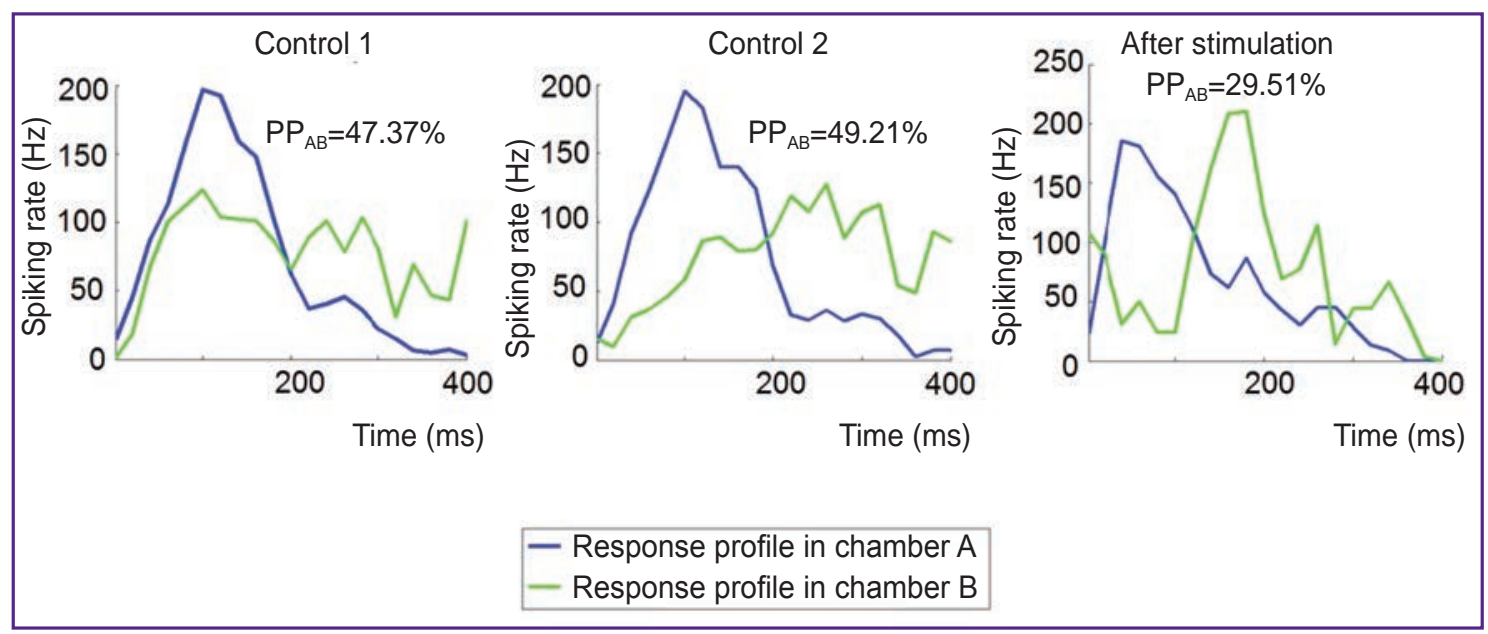

Figure 5. An example of depression after tetanic stimulation of presynaptic and postsynaptic neurons in the network; $\mathrm{PP}_{\mathrm{AB}}$ value declined significantly only after tetanic stimulation

therefore, culture sites in the area located in close proximity to microchannels were selected as sites with pre- and postsynaptic neurons: a presynaptic segment in chamber $A$, a postsynaptic segment in chamber $B$. In tetanic stimulation, 4 electrodes in chamber $A$ and 4 electrodes in chamber $B$ were stimulated with a delay of $10 \mathrm{~ms}$. Chamber A was stimulated first (see "Materials and Methods") to induce STDP plasticity in the excitatory synapses of hippocampal cells [23, 24].

Potentiation. The number of stimuli which evoked response in the form of network bursts was calculated separately for chambers A and B. We estimated propagation probability $\mathrm{PP}_{\mathrm{AB}}$ as a percentage of bursts evoked in the source chamber which, in turn, evoked bursts in the receiver chamber of total number of bursts induced by stimulation in the source chamber. This parameter indicates the efficiency of bioelectrical signal transmission between pre- and postsynaptic subpopulations of neural cells.

$\mathrm{PP}_{\mathrm{AB}}$ was evaluated independently for each stimulating electrode. $\mathrm{PP}_{\mathrm{AB}}$ for one of the stimulating electrodes in two sequences of control stimulation and after tetanic stimulation was found to be 90.2, 83.7 and $96.7 \%$, respectively. In other words, spontaneous change amounted to $7.3 \%$ (the difference between $\mathrm{PP}_{\mathrm{AB}}$ in the second and the first control stimulation), while the induced change was quite significant at $15.5 \%$ (the difference between $\mathrm{PP}_{\mathrm{AB}}$ after high-frequency stimulation and $\mathrm{PP}_{\mathrm{AB}}$ in the second control stimulation). An example of induced changes is shown in Figure 4. High frequency tetanic stimulation induced potentiation of functional connectivity between two subnetworks. Such effect of connectivity potentiation was expected after applying the stimulation protocol first for presynaptic neurons and next for postsynaptic ones in accordance with STDP effect [23].

Depression. In experiments on other cultures, we observed depression of synaptic pathways, $\mathrm{PP}_{\mathrm{AB}}$ value of responses to stimuli in chamber A decreased after tetanic stimulation (Figure 5). In two control stimulation sequences and after tetanic stimulation it measured 47.37, 49.21 and 29.51\%, respectively. In other words, spontaneous change measured $3.9 \%$ (the difference between the first and second control stimulation), the induced change equaled $40.0 \%$ (the difference between responses after high frequency stimulation and the second control stimulation).

Only one of the 5 cultures showed increase in $\mathrm{PP}_{\mathrm{AB}}$ value after high-frequency stimulation, suggesting connectivity potentiation (Figure 6). Decrease in $\mathrm{PP}_{\mathrm{AB}}$ value was observed in three cultures. In one culture, spontaneous $\mathrm{PP}_{\mathrm{AB}}$ fluctuations were proportionate to changes in $\mathrm{PP}_{\mathrm{AB}}$ after high frequency stimulation for all stimulating electrodes.

Types of neural network responses to stimuli. During the analysis it was found that responses to stimuli consisted of two different types of activity depending on the total number of spikes evoked in the bursts (Figure 7). Such network activity with two types of bursts was found separately in chambers $A$ and $B$. We divided responses into two clusters: big and small responses, using K-means clustering algorithm. The analysis was performed for each subnetwork in chambers $A$ and $B$ (Figure 7 (a)-(c) and (d)-(f)). It is noteworthy that the two types of responses (see Figure 7 (b) and (c), (e) and (f)) differed significantly in spiking rate profile (number of spikes in each 20-ms time interval).

Next, we analyzed how different types of responses (big and small) reflected changes in the network (plasticity) induced by high-frequency tetanic stimulation using the example of cultures where increase and decrease in $\mathrm{PP}_{\mathrm{AB}}$ values was observed. For big responses, $\mathrm{PP}_{\mathrm{AB}}$ values measured 27.27, 21.43 and $44.19 \%$ during the first two control stimulation sequences and after tetanic stimulation. In other words, a spontaneous change in probability of the evoked burst 


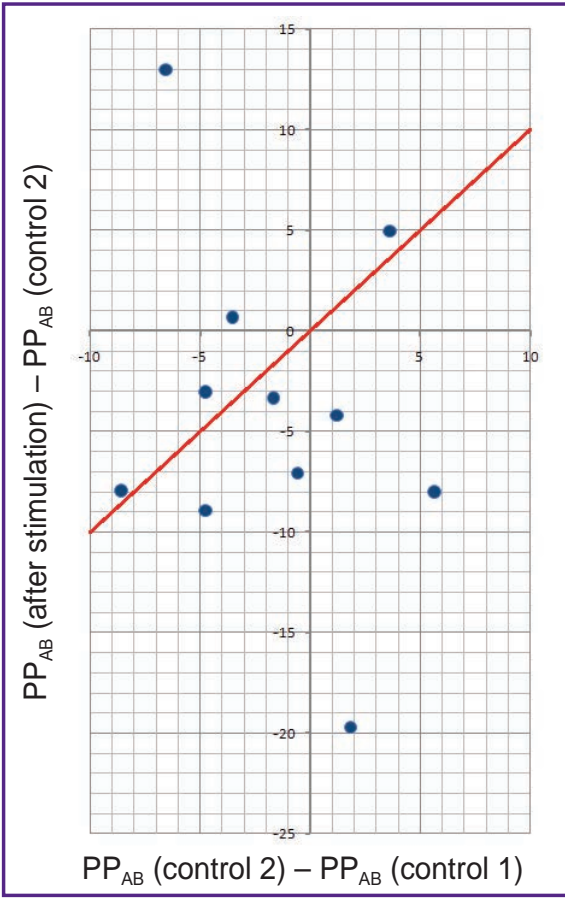

Figure 6. The effect of high frequency stimulation of two cell populations with $10 \mathrm{~ms}$ delay on percentage of evoked responses transmitted from presynaptic to postsynaptic subnetwork $\left(\mathrm{PP}_{\mathrm{AB}}\right)$

One point corresponds to one stimulating electrode in chamber A (in total, 11 electrodes, 5 cultures). The red line indicates the values at which spontaneous $\mathrm{PP}_{\mathrm{AB}}$ fluctuation is equal to $\mathrm{PP}_{\mathrm{AB}}$ change after high frequency stimulation

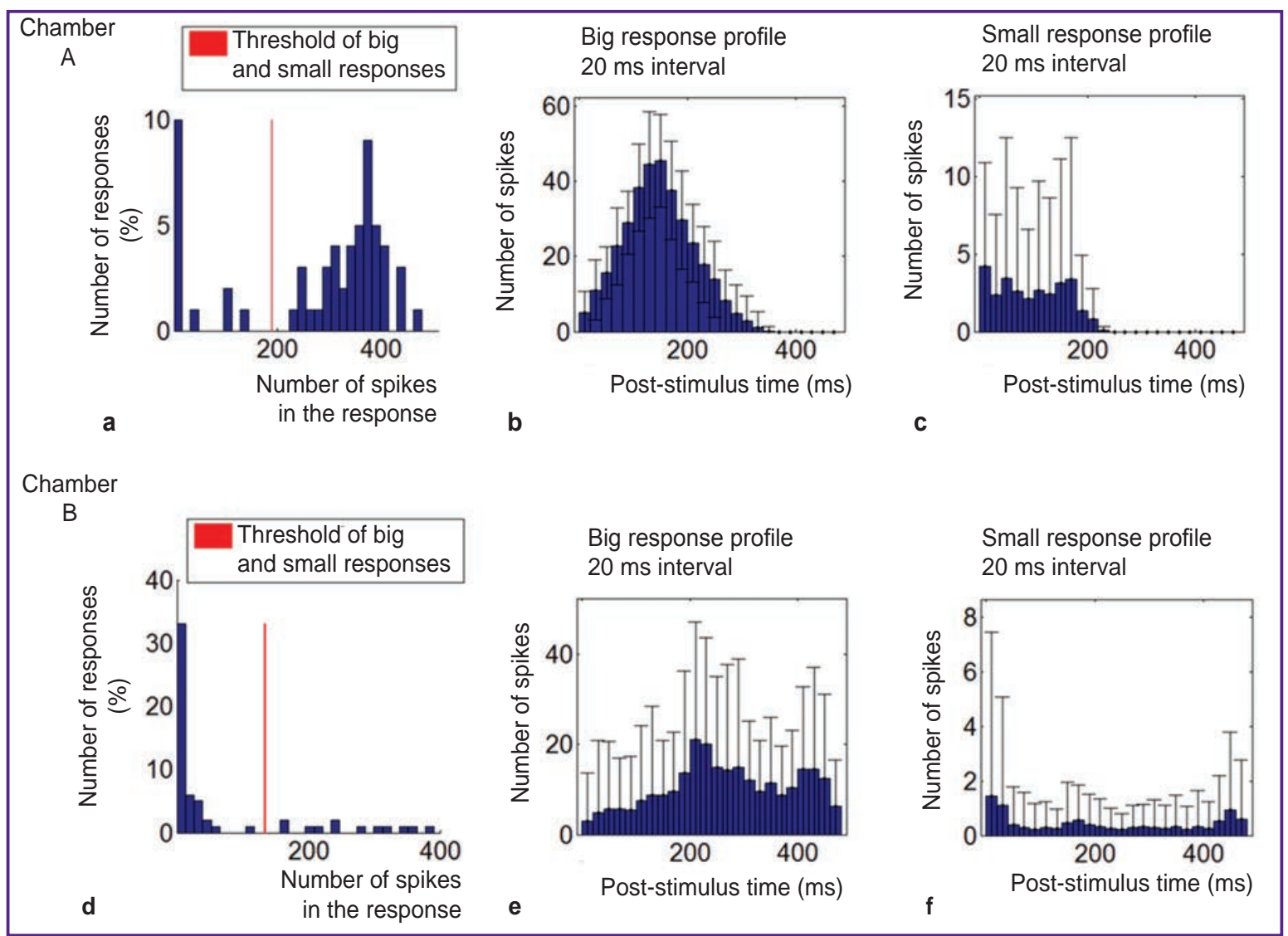

Figure 7. Two types of cultured neural network responses to low frequency stimuli through the selected electrode located in chamber A:

(a) distribution of responses to stimuli in chamber A depending on the total number of spikes in the response; the red line shows the threshold for identifying big and small responses; (b) histograms of post-stimulus network activity for big network responses in chamber A (PSTH A); (c) PSTH A for small responses; (d) distribution of responses to stimuli in chamber $B$, depending on the total number of spikes in the response; the red line shows the threshold for identifying big and small responses; (e) histograms of post-stimulus network activity for big responses from the network in chamber B (PSTH B); (f) PSTH B for small responses 


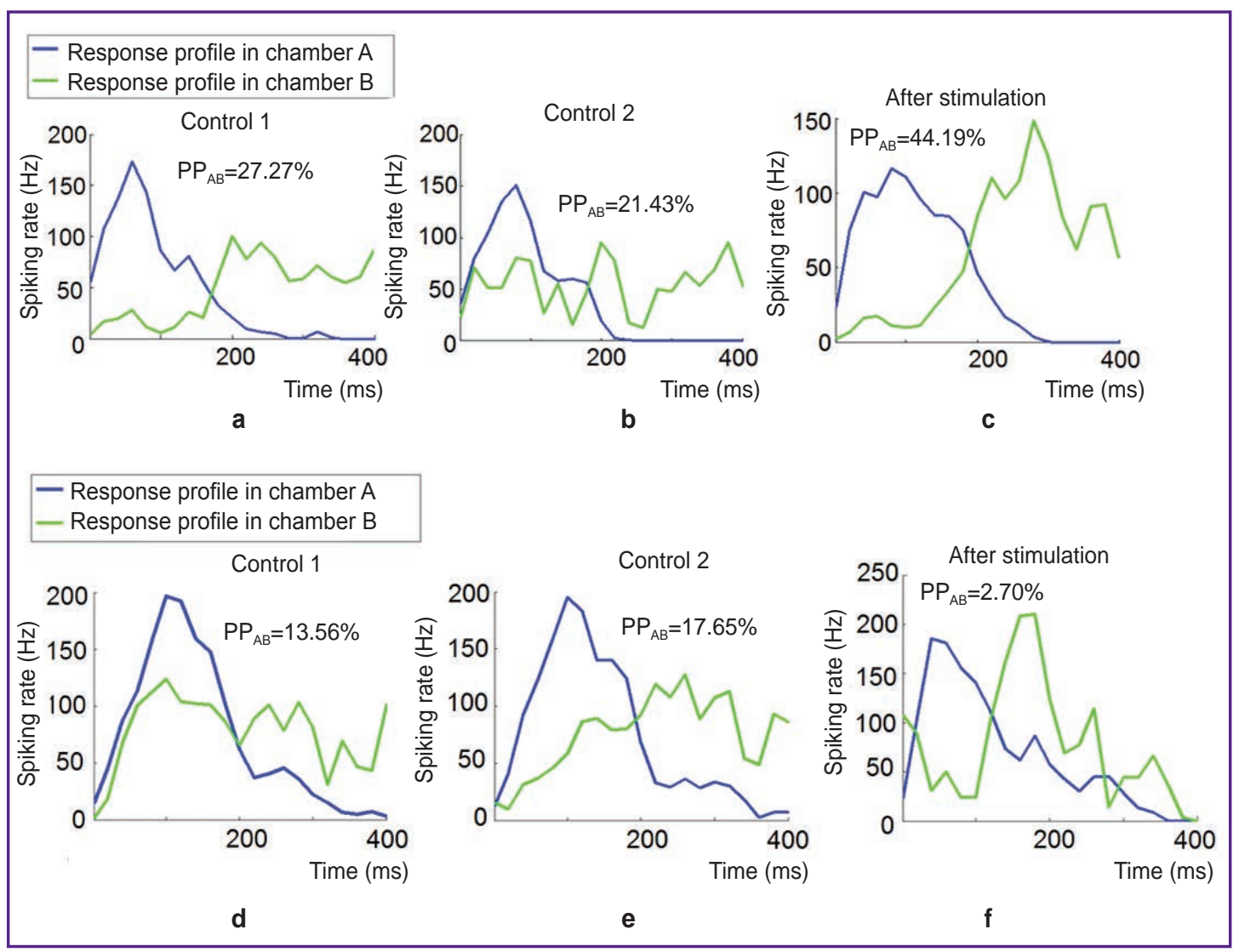

Figure 8. Clusters of big responses (a)-(c) and small responses (d)-(f) in separate chambers:

Profiles of responses to stimuli in presynaptic chamber are in blue, while in postsynaptic chamber they are green; figures (a), (b), (d), (e) represent responses after two control stimulation sequences, diagrams (c), (f) show responses after tetanic stimulation

propagation between chambers (the difference between the first and second control stimulation) was $21.42 \%$, while the change induced by high-frequency stimulation (the difference between responses after high frequency stimulation and the second control stimulation) was $106 \%$ for big responses. $\mathrm{PP}_{\mathrm{AB}}$ values for small responses were 0 and $22.2 \%$ in control stimulation sequences and $33.3 \%$ after high frequency stimulation. We considered such data as unstable activity, which provides no possibility of estimating the effect. Comparison of $\mathrm{PP}_{\mathrm{AB}}$ values for big and small responses in separate chambers is shown in Figure 8.

Discussion. In this paper, we proposed a new method of studying synaptic plasticity in dissociated cultured neural networks grown on MEA with a developed microfluidic chip. The chip consists of two chambers connected by special microchannels which stimulate a unidirectional axonal growth between the chambers and ensure a certain control over synaptic pathways in the culture. Synaptic plasticity effects have previously been studied in the networks of neuronal cultures with homogeneous architecture of synaptic network [9, 10]. For this purpose, we applied a previously developed method and technology for growing axonal branches through microchannels between two cultures of hippocampal neurons [22] and now we are the first to present a method of studying synaptic plasticity in heterogeneous network architecture with a specific arrangement of presynaptic and postsynaptic neurons (see Figure 1 (d)).

To study synaptic plasticity, we applied tetanic stimulation with a $10 \mathrm{~ms}$ delay for two groups of electrodes in chambers $A$ and $B$ in accordance with plasticity induction protocol STDP [23, 24]. The characteristics of stimulus-induced network burst propagation between pre- and postsynaptic neuronal populations were found to change significantly after a high-frequency tetanic stimulation with a $10 \mathrm{~ms}$ delay (see Figures 5-8). In one culture of the five used, the stimulation induced potentiation of synaptic pathways in microchannels leading to increase in probability of burst propagation between chambers. Surprisingly, in the other three cultures, we found the effect of stimulus induced activity depression after tetanic stimulation. The cases of decrease in probability of network signal propagation between populations observed in the experiments can be associated with the fact that some neurons in hippocampal cell culture are inhibitory (about 
$20 \%)[25,26]$. When connections of inhibitory neurons are stimulated with $\mathrm{a} \pm 10 \mathrm{~ms}$ time delay, increase in the inhibitory effect is observed [27]. Reduced functional connectivity might be associated with neurotransmitter depletion after high frequency stimulation or the property of homeostatic plasticity.

These experiments showed effectiveness of the developed experimental model to induce functional changes in cultured neural networks.

Conclusion. The proposed experimental method for studying synaptic plasticity provides a reliable control of cultured network morphology to induce spike timing dependent plasticity. This approach can be used to study network effects of synaptic plasticity in the processes of memory, learning, information processing and brain tissue recovery.

Acknowledgements. The authors thank Eugene Malishev for his valuable contribution to the development of microfluidic chips, master mold fabrication, methodology optimization and discussion of research results.

Study Funding. This study was supported by a grant from the President of the Russian Federation (project MK-8731.2016.4).

Conflict of Interests. The authors have no conflict of interests to disclose.

\section{References}

1. Marom S., Shahaf G. Development, learning and memory in large random networks of cortical neurons: lessons beyond anatomy. Q Rev Biophys 2002; 35(01): 63-87, https:// doi.org/10.1017/s0033583501003742.

2. Le Feber J., Stegenga J., Rutten W.L.C. The effect of slow electrical stimuli to achieve learning in cultured networks of rat cortical neurons. PLoS One 2010; 5(1): e8871, https:// doi.org/10.1371/journal.pone.0008871.

3. Pimashkin A., Gladkov A., Mukhina I., Kazantsev V. Adaptive enhancement of learning protocol in hippocampal cultured networks grown on multielectrode arrays. Front Neural Circuits 2013; 7: 87, https://doi.org/10.3389/fncir.2013.00087.

4. Li Y., Zhou W., Li X., Zeng S., Luo Q. Dynamics of learning in cultured neuronal networks with antagonists of glutamate receptors. Biophys J 2007; 93(12): 4151-4158, https://doi.org/10.1529/biophysj.107.111153.

5. Bologna L.L., Nieus T., Tedesco M., Chiappalone M., Benfenati F., Martinoia S. Low-frequency stimulation enhances burst activity in cortical cultures during development Neuroscience 2010; 165(3): 692-704, https://doi.org/10.1016/j. neuroscience.2009.11.018.

6. Brewer G.J., Boehler M.D., Ide A.N., Wheeler B.C. Chronic electrical stimulation of cultured hippocampal networks increases spontaneous spike rates. J Neurosci Methods 2009; 184(1): 104-109, https://doi.org/10.1016/j. jneumeth.2009.07.031.

7. Jimbo Y., Robinson H.P.C., Kawana A. Strengthening of synchronized activity by tetanic stimulation in cortical cultures: application of planar electrode arrays. IEEE Trans Biomed Eng 1998; 45(11): 1297-1304, https://doi.org/10.1109/10.725326.

8. Jimbo Y., Tateno T., Robinson H.P.C. Simultaneous induction of pathway-specific potentiation and depression in networks of cortical neurons. Biophys J 1999; 76(2): 670-678, https://doi.org/10.1016/s0006-3495(99)77234-6.

9. Wagenaar D.A., Pine J., Potter S.M. Searching for plasticity in dissociated cortical cultures on multi-electrode arrays. J Negat Results Biomed 2006; 5(1): 16, https://doi. org/10.1186/1477-5751-5-16

10. Chiappalone M., Massobrio P., Martinoia S. Network plasticity in cortical assemblies. Eur J Neurosci 2008; 28(1): 221-237, https://doi.org/10.1111/j.1460-9568.2008.06259.x.

11. Chiappalone M., Bove M., Vato A., Tedesco M., Martinoia S. Dissociated cortical networks show spontaneously correlated activity patterns during in vitro development Brain Res 2006; 1093(1): 41-53, https://doi.org/10.1016/j. brainres.2006.03.049.

12. Feber J. le, Rutten W.L.C., Stegenga J., Wolters P.S., Ramakers G.J.A., Pelt J. van. Conditional firing probabilities in cultured neuronal networks: a stable underlying structure in widely varying spontaneous activity patterns. J Neural Eng 2007; 4(2): 54-67, https://doi.org/10.1088/17412560/4/2/006.

13. Taylor A.M., Blurton-Jones M., Rhee S.W., Cribbs D.H., Cotman C.W., Jeon N.L. A microfluidic culture platform for CNS axonal injury, regeneration and transport. Nat Methods 2005; 2(8): 599-605, https://doi.org/10.1038/nmeth777.

14. Kanagasabapathi T.T., Ciliberti D., Martinoia S., Wadman W.J., Decré M.M. Dual-compartment neurofluidic system for electrophysiological measurements in physically segregated and functionally connected neuronal cell culture. Front Neuroeng 2011; 4: 13, https://doi.org/10.3389/ fneng.2011.00013.

15. Takayama Y., Kotake N., Haga T., Suzuki T., Mabuchi K. Formation of one-way-structured cultured neuronal networks in microfluidic devices combining with micropatterning techniques. J Biosci Bioeng 2012; 114(1): 92-95, https://doi. org/10.1016/j.jbiosc.2012.02.011.

16. Bisio M., Bosca A., Pasquale V., Berdondini L., Chiappalone M. Emergence of bursting activity in connected neuronal sub-populations. PLoS One 2014; 9(9): e107400, https://doi.org/10.1371/journal.pone.0107400.

17. Le Feber J., Postma W., de Weerd E., Weusthof M., Rutten W.L.C. Barbed channels enhance unidirectional connectivity between neuronal networks cultured on multi electrode arrays. Front Neurosci 2015; 9: 412, https://doi. org/10.3389/fnins.2015.00412.

18. Pan L., Alagapan S., Franca E., Leondopulos S.S., DeMarse T.B., Brewer G.J., Wheeler B.C. An in vitro method to manipulate the direction and functional strength between neural populations. Front Neural Circuits 2015; 9: 32, https:// doi.org/10.3389/fncir.2015.00032.

19. Yoshizumi Y., Honegger T., Berton K., Suzuki H., Peyrade D. Micromotors: trajectory control of self-propelled micromotors using AC electrokinetics (Small 42/2015). Small 2015; 11(42): 5629-5629, https://doi.org/10.1002/ smll.201570255.

20. Habibey R., Golabchi A., Latifi S., Difato F., Blau A. A microchannel device tailored to laser axotomy and longterm microelectrode array electrophysiology of functional regeneration. Lab Chip 2015; 15(24): 4578-4590, https://doi. org/10.1039/c5lc01027f.

21. Pimashkin A., Kastalskiy I., Simonov A., Koryagina E., Mukhina I., Kazantsev V. Spiking signatures of spontaneous activity bursts in hippocampal cultures. Front Comput Neurosci 2011; 5: 46, https://doi.org/10.3389/fncom.2011.00046. 
22. Malishev E., Pimashkin A., Gladkov A., Pigareva Y., Bukatin A., Kazantsev V., Mukhina I., Dubina M. Microfluidic device for unidirectional axon growth. J Phys Condens Matter 2015; 643: 01202, https://doi.org/10.1088/17426596/643/1/012025.

23. Bi G.Q., Poo M.M. Synaptic modifications in cultured hippocampal neurons: dependence on spike timing, synaptic strength, and postsynaptic cell type. J Neurosci 1998; 18(24): 10464-10472.

24. Debanne D., Gahwiler B.H., Thompson S.M. Asynchronous pre- and postsynaptic activity induces associative long-term depression in area CA1 of the rat hippocampus in vitro. Proc Natl Acad Sci USA 1994; 91(3): 1148-1152, https://doi.org/10.1073/pnas.91.3.1148.
25. Woodin M.A., Ganguly K., Poo M. Coincident preand postsynaptic activity modifies gabaergic synapses by postsynaptic changes in $\mathrm{Cl}^{-}$transporter activity. Neuron 2003; 39(5): 807-820, https://doi.org/10.1016/s0896-6273 (03)00507-5.

26. Chen X., Dzakpasu R. Observed network dynamics from altering the balance between excitatory and inhibitory neurons in cultured networks. Phys Rev E Stat Nonlin Soft Matter Phys 2010; 82(3): 031907, https://doi.org/10.1103/ physreve.82.031907.

27. Jinno S., Kosaka T. Stereological estimation of numerical densities of glutamatergic principal neurons in the mouse hippocampus. Hippocampus 2010; 20(7): 829-840, https://doi.org/10.1002/hipo.20685. 